\title{
Editorial
}

\section{Order of authorship: A call for more transparency}

All academics know that discussions about the order of authorship for a published paper can be quite complicated and even fraught. Who is the first author? What is the sequence for the others? Should all members of a research team be included, even if they have contributed little to the drafting of a paper? And, where scholarly careers are shaped by the ability to publish, these decisions become critical. These discussions are sometimes resolved easily, but they may also result in serious conflict when authors feel that their contributions to a paper are not properly acknowledged.

Discussions about the order of authorship are complicated as there are no clear standards for the sequence of authors, especially for journals like Information Polity, which publishes articles by authors from different disciplinary backgrounds. In some disciplines, the norm is to put authors in alphabetical order, whereas in others, the first named author is the key author or the most senior academic involved in the publication. The final author listed in a publication in some disciplines is regarded as the research group leader, but in others seen as the academic who contributed least to the research presented in the publication.

At Information Polity, we have never imposed a single norm on the sequence of authors for published articles. We have always left it to the team of authors to discuss and decide on this sequence themselves. At the same time, this policy feels a little uncomfortable as it means that we publish papers for which the order of authorship may have very different meanings to different scholars. Our readers cannot tell what the order of authorship actually means about the contributions of the different authors involved.

Many journals are now implementing policies that require the disclosure of individual authors' contributions (Sauermann \& Haeussler, 2017) to individual manuscripts and the research on which they are based. Here, the intention is to create greater transparency, clarity and fairness. Whilst at Information Polity we have not imposed such a norm, we are encouraging our authors to indicate briefly what the order of authorship for their paper means. In this respect, we are asking authors to provide clarity about the reasons for the order of authorship. There are a number of possible options (without pretending to be exhaustive):

- Authors could indicate that the order of authorship is alphabetical or even random since all authors contributed equally to the paper;

- Authors can indicate that the order reflects the extent of the contribution of each author, with the first author having contributed most and the last the least;

- Authors can describe the specific contribution of each author to the paper. For example, author $\mathrm{X}$ coordinated the paper and contributed to all parts of it, author $\mathrm{Y}$ contributed to the theoretical framework and the data analysis and author $\mathrm{Z}$ contributed to the empirical data collection; or

- Authors can explain that one author was a doctoral student and the other the principal supervisor. 
These clarifications can provide more transparency to the readers and they can also help to reduce conflict between authors by properly acknowledging their different contributions. These forms of transparency can also help to reduce 'honorary or ghost authors' (Dotson \& Slaughter, 2011) who are mentioned as authors but did not provide any substantial contribution to the final published work. Finally, for doctoral students who publish papers with their supervisors, which is the norm in some disciplines, this form of transparency may help to clarify their key role to their $\mathrm{PhD}$ assessment committee.

Clearly, there is a need for greater transparency about the order of authorship in published work, and over time disciplinary norms are emerging. Whilst we do not assume that the form of transparency encouraged above eliminates all conflict about this sensitive issue, it may result in a beneficial debate amongst authors about the way they collaborate on papers. In turn, these forms of transparency help to establish constructive forms of team science that are required to advance research in our field.

\author{
Professor Albert Meijer \\ E-mail: A.J.Meijer@uu.nl \\ Professor William Webster \\ E-mail: william.webster@stir.ac.uk \\ Editors-in-Chief
}

\title{
References
}

Dotson, B., \& Slaughter, R. L. (2011). Prevalence of articles with honorary and ghost authors in three pharmacy journals. American Journal of Health-System Pharmacy, 68(18), 1730-1734.

Sauermann, H., \& Haeussler, C. (2017). Authorship and contribution disclosures. Science Advances, 3(11), e1700404. 